\title{
NUEVO LIDERAZGO ORGANIZACIONAL PARA FORTALECER INSTITUCIONES UNIVERSITARIAS DÉBILMENTE ACOPLADAS SEGÚN WEICK
}

\author{
Waldyr Fong Silva ${ }^{1 *}$, Arnulfo Tarón Dunoyer ${ }^{2}$, Randy Zabaleta Mesino $^{3}$ \\ 1,2, Universidad de Cartagena. Cartagena de Indias, Colombia \\ ${ }^{3}$ Universidad Nacional Abierta y a Distancia-UNAD. Cartagena de Indias, Colombia \\ *Autor de correspondencia: wfongs@ unicartagena.edu.co
}

Recibido Octubre 2019; Aceptado Diciembre 2019

\begin{abstract}
Resúmen - En el presente trabajo se hace un análisis de la institución universitaria del siglo XXI desde la perspectiva de weick (1976) y como el débil acoplamiento entre sus estamentos inciden en sus procesos organizacionales desde la alta dirección hacia las unidades académicas y departamentos. Se concluye que la flexibilidad en el funcionamiento de las universidades modernas se garantiza precisamente por la débil articulación de sus elementos constituyentes que potencializan su efectividad por el alto índice de iniciativa con el que cuentan en el modelo universitario y a la capacidad de solicitar colaboración de cualquiera de las demás unidades universitarias, lo que permite identificar que el sistema jerárquico Universitario es no lineal. Así mismo unificar el liderazgo transaccional y transformacional universitario en un solo liderazgo como el liderazgo directivo con características del nuevo liderazgo de Burns, permitirá transformar la organización universitaria en Meta-Universidad con altos estándares de calidad.
\end{abstract}

\section{NEW ORGANIZATIONAL LEADERSHIP TO STRENGTHEN WEAKLY COUPLED COLLEGE INSTITUTIONS ACCORDING TO WEICK}

Abstract - In this paper, an analysis of the university institution of the XXI century is made from the perspective of Weick (1976) and how the weak coupling between its estates affects its organizational processes from the top management to the academic units and departments. It is concluded that the flexibility in the functioning of modern universities is guaranteed precisely by the weak articulation of its constituent elements that potentiate their effectiveness due to the high index of initiative they have in the university model and the ability to request collaboration from any of the other university units, which allows us to identify that the University hierarchical system is non-linear. Likewise, unifying the transactional and transformational university leadership in a single leadership as the directive leadership with characteristics of the new Burns leadership, will allow to transform the university organization into Meta-University with high quality standards.

Keywords: Weick, leadership, organization, weak coupling, university.

IPSA SCIENTIA - Vol. 4 № 1 - 2019 


\section{Introducción}

\section{LA UNIVERSIDAD CONCEBIDA DESDE EL ENFOQUE DE WEICK}

La universidad puede concebirse desde el enfoque de lo que Weick (1976) denominó estructuras débilmente acopladas o articuladas, con el objeto de que se transforme en META-UNIVERSIDAD (Graves. 1997, Pág. 97). La flexibilidad en el funcionamiento de las universidades modernas se garantizan precisamente por la débil articulación de sus elementos constituyentes que potencializan su efectividad gracias al alto índice de iniciativa con el que cuentan en el modelo universitario y a la capacidad de solicitar colaboración de cualquiera de las demás unidades universitarias, caracterizándose estructuralmente por la "NO" linealidad de su sistema jerárquico y verificándose cada una de las características de las estructuras débilmente acopladas (Weick, 1976). En estas organizaciones las líneas de mando no se encuentran bien definidas lo que genera cierta "flexibilidad y debilidad administrativa" ya que la línea de mando no es horizontal a pesar de encontrarse conformadas por vicerrectorías, unidades académicas, departamentos, oficinas asesoras entre otras dependencias, articuladas o acopladas entre sí pero gozando de cierto grado de independencia, autonomía e identidad con características de autogobierno. Por esta razón, es que podemos decir que son organizaciones débilmente acopladas pero que hasta cierto punto -en ocasiones- se convierten en una fortaleza (Weick, 1976).

A continuación en la Figura 1 se puede observar un modelo "no linealizado" desde la perspectiva de Weick de una estructura universitaria débilmente acoplada en donde podemos apreciar líneas curvas que simbolizan la debilidad del acoplamiento existente en las universidades así como también de la no horizontalidad en la cadena ejecutiva de mando de cada organismo. Así mismo, representan la simbiosis que se presenta día a día en cada uno de sus elementos organizacionales y de control universitario. Estos aspectos impactan en la forma como se relaciona la Universidad con su entorno, es decir, la forma como interactúa "débilmente" si se puede decir así, con los diferentes sectores de su desempeño institucional como lo son la comunidad, sector empresarial, gubernamental e impacto cuando se trabajan procesos de innovación y desarrollo tecnológico (Weick, 1976; Didriksson, 2004)

Figura No.1. Organización Universitaria desde la perspectiva de Weick con elementos débilmente acoplados.

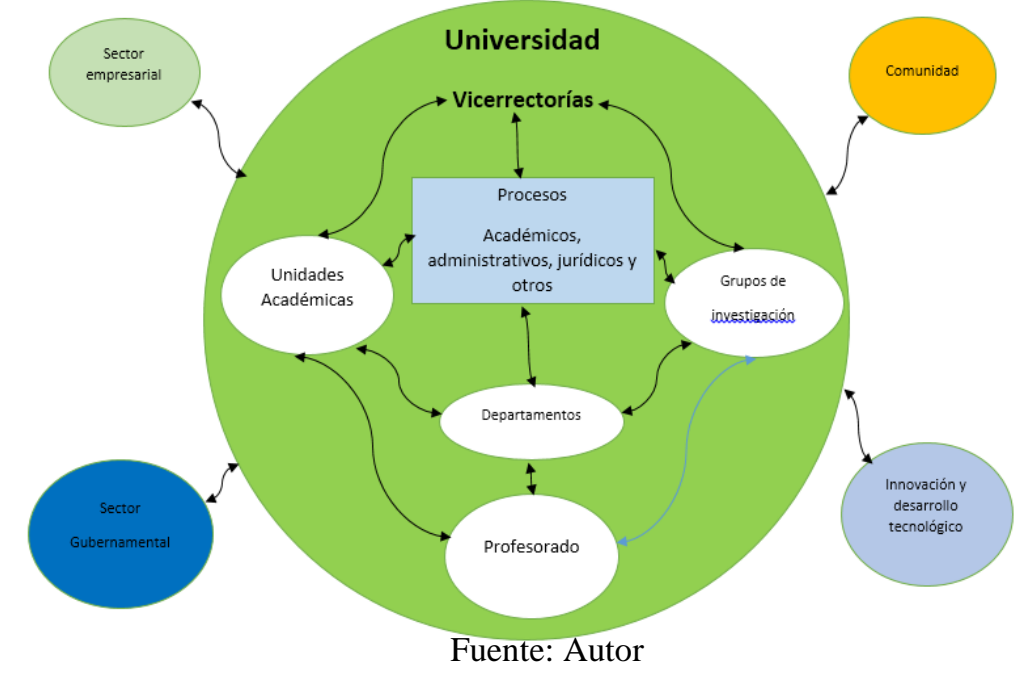

IPSA SCIENTIA - Vol. 4 № 1 - 2019 
De la misma manera en la Figura 1 se resaltan las unidades académicas, departamentos, grupos de investigación, profesorado, vicerrectorado y Universidad caracterizados por su inter-relación continua al interior de la Universidad y a la vez con los sectores de impacto como el empresarial, gubernamental y comunitario mediados por los procesos de apropiación social del conocimiento y procesos de innovación y desarrollo tecnológico (Fong et al., 2017; Fong et al., 2018). Todos estos estamentos al interior de la Universidad establecen una interacción continua no horizontal con todos los demás de la comunidad académica tanto de tipo administrativo como de gestión (facultades, institutos universitarios, otros departamentos, entre otros). Por estas razones, todas las relaciones entre los diferentes estamentos universitarios tanto al interior como al exterior de la Universidad se dice que se encuentran débilmente acoplados desde la perspectiva de Weick (1976).

\section{CARACTERÍSTICAS DE LAS ESTRUCTURAS DÉBILMENTE ACOPLADAS SEGÚN WEICK:}

\section{a) Autonomía}

Se verifica en los siguientes aspectos: a) Organización académica (proyecto docente, grupos estudiantiles, planes de estudio, entre otros) y b) Gestión administrativa y de personal (reclutamiento y promoción de personal) (La Rotta et al., 2020). Proporciona a las unidades básicas, miembros y unidades organizativas las herramientas y estrategias que le permiten alcanzar objetivos propuestos (a corto, mediano y largo plazo) y en relación con los dos rasgos característicos de las estructuras débilmente acopladas como lo son la ambigüedad de metas y la ausencia de mecanismos efectivos de control.

\section{b) Ambigüedad de metas}

Las metas establecidas en la institución universitaria se encuentra organizada en el plan de desarrollo institucional direccionado por el liderazgo directivo y los diferentes puntos de vista, opinión, posición, toma de decisiones, línea de actuación o conducta ocasionando lo más común que es la ambigüedad de metas en virtud de la autonomía de cada una de las partes de la organización universitaria (Linares \$ Geizzelez, 2016; Wall et al, 2004). Esto se extrapola a cada dependencia de la universidad ya que cada una se considera con metas independientes y consideraciones propias ajenas a la misión y plan de desarrollo de la misma universidad (Waddock \& Graves, 1997a; 1997b).

\section{c) Ausencia de Mecanismos efectivos de control}

El control de los procesos académico-administrativos universitarios constituye uno de los principales inconvenientes en materia de control ya que la evaluación -por su carácter- persigue diagnosticar para luego intervenir en los procesos con ánimo de plantear y proponer planes de control y mejora continua según los criterios establecidos en los sistemas de gestión de calidad universitarios (Uglade, 1987). A parte del formalismo de control establecido por la universidad, la otra figura de control la constituye el que hacen los docentes (condicionado por la autonomía y el celularismo manifiesto en sus dependencias) y administrativos referidos al control de la presencia física de los docentes en sus puestos de trabajo y cuyo

IPSA SCIENTIA - Vol. 4 № 1 - 2019 
cumplimiento sólo depende de su profesionalismo y honestidad. Por otro lado, los procesos y decisiones de los organismos de control universitario se encuentran influenciados por el amiguismo y sesgamiento profesional interno en donde la independencia no es propia y por el contrario, la mayoría de sus decisiones son de tipo político y no amparadas en el ordenamiento jurídico sino en apreciaciones personales de quienes ostentan altos cargos en la dirección universitaria aspectos estos que la hacen débilmente acopladas según los criterios de Weick (1976) y Mejías (2013).

\section{INSTITUTOS COMO CULTURAS BALCANIZADAS}

Los institutos son Subgrupos fuertemente aislados entre sí en la institución universitaria, claramente delimitados en el espacio y poseen permanencia duradera, son estables, perduran en el tiempo y no se consideran docentes en general (Hernández, 2013). Es decir, los miembros de este organismo se consideran avanzados con respecto al resto de miembros docentes de la institución ya sea por su nivel académico (doctorado y post-doctorado) como por sus procesos de investigación, innovación y desarrollo tecnológico que adelantan y que los han posicionado como "los mejores" de la universidad (Fong et al., 2018; Morioka et al., 2018). Sin embargo, su celularismo y aislamiento impactan negativamente en las metas y visión institucional debido a su débil acoplamiento con la organización así como lo platea Weick (1976).

Por otro lado, la cultura universitaria es la encargada de orientar las acciones organizativas, la solución de problemas, la acción pedagógica entre otras más desde su marco de pensamiento y esquemas de conducta de manera que la integración exitosa de nuevos miembros a la institución universitaria se encuentra condicionada por la cultura y normativa existente que se constituyen en el soporte de toda relación y decisión al interior de cada una de las unidades académicas básicas (Muñoz et al., 2018). Uno de los principales inconvenientes -desde hace mucho tiempo en la universidad- radica en el choque de poderes entre integrantes de áreas de conocimiento afines y que se genera al interior de las mismas debido a los intereses profesionales y corporativos de cada uno de sus miembros (docentes, directivos, trabajadores y administrativos) debido a su débil acoplamiento según Weick (1967). Esto ocasiona que los integrantes de cada grupo actúen de manera aislada impidiendo el debate científico y el desarrollo institucional (Orton \& Weick, 1990). Esto significa además, que la realidad científica y académica universitaria se encuentre -en gran medida- direccionada por la realidad administrativa de la institución y no como debería ser, es decir, sustentada en soportes científicos con altos valores de independencia y autonomía hacia metas comunes y consensuadas de pie hacia la Meta-Universidad (Graves. 1997, Pág. 97).

\section{UNIVERSIDAD COMO ENCUENTRO POLÍTICO}

En las universidades debido a la autonomía de las partes y a su estructura débilmente acoplada, el poder se desplaza o sesga en gran medida hacia las unidades básicas o base (programas, departamentos, dependencias) constituyéndose esto en una preocupación de primer nivel ya que la política que se genera en ellas se lleva a cabo a través de mecanismos conocidos como lo son la negociación, uso y manejo de la información, búsqueda de consenso, establecimiento de alianzas, coaliciones entre otras estrategias,

IPSA SCIENTIA - Vol. 4 № $1-2019$ 
trayendo como consecuencia lo siguiente: a) Dispersión del poder organizacional ocasionando que difícilmente pueda ser controlado y b) duración de conflictos de manera duradera debido a los esfuerzos que los miembros de la organización realizan buscando aliados y refuerzos para la mejora de sus intereses (Salazar, 2006; Siboni et al., 2013). Esto genera el mismo "ciclo débilmente acoplado" en los demás miembros de la organización de manera que se retroalimenta el mismo una y otra vez. De esta manera, se consolida la "arena política" mediante lo que se conoce como "mecanismos perversos de socialización y acción ya que los nuevos miembros se unen a uno de los grupos que en el momento están al mando haciendo valer sus intereses y en donde "la política" es la única motivación admisible para las acciones de cada uno de sus miembros, ocasionando con esto, la agudización del régimen débilmente acoplado en donde se terminan tratando hasta los asuntos más mínimos y aparentemente de menos importancia en las unidades básicas de la organización (Pons \& Ramos, 2012).

\section{INTER-RELACIÓN EN LOS SISTEMAS DE LA ORGANIZACIÓN}

La institución universitaria debe entenderse como un sistema social complejo con acoplamiento débil, celularista y con alto grado de autonomía entre sus partes, es decir, además de contemplar aspectos objetivos, también contempla aspectos subjetivos y culturales que hacen referencia a relaciones entre las unidades básicas de la organización (Gómez, 2008). Así mismo contempla aspectos relacionados con las creencias, intereses, expectativas y formas de hacer el trabajo, ocasionando en las personas diversas formas de percibir su misión en la Universidad. Este acontecer diario del ser humano en las Universidades se desarrolla en una trama de relaciones y de sucesos orientados por las instituciones y que pasan a interiorizarse constituyéndose en canalizadores y reguladores sociales internos (Harris, 2012). Esta actividad interna de relaciones en la institución universitaria se desarrolla direccionada por tres (3) parámetros fundamentales como lo son: a) docencia b) investigación y c) extensión y proyección social.

Por otro lado, el rol del docente universitario se desenvuelve en una gran trama de relaciones sociales también débilmente acopladas que se desarrollan a través de una amplia y compleja red de comunicaciones en la que la preparación técnica del recurso humano docente y administrativo y su disposición personal para afrontar situaciones externas e internas es fundamental (Orton y Weick, 1990; Maureira et al., 2014).

Otra de las razones a las que hay que prestar atención en las instituciones universitarias en cuanto al sistema de relaciones sociales débilmente acopladas según Weick (1976) son: a) práctica docente centrada en el profesor aspecto que aún se disemina con la autonomía docente b) profesores con funciones de multiacoplamiento débil, caracterizándose por contemplar funciones tripartitas de docencia, investigación y extensión y proyección social) c) capacidad de integración en el sistema universitario débilmente acoplado que permita su acoplamiento al clima organizacional universitario mediante mecanismos eficaces de comunicación y asertividad d) debilidad en la formación del profesorado impide su adecuado acoplamiento en relaciones sociales, progreso y desarrollo que impacten en los procesos comunicacionales de la organización e) la autonomía profesional se relaciona en alto grado con el ambiente institucional, sin embargo, se considera débilmente acoplada con las metas institucionales debido al alto grado de celularismo de este grupo de profesionales (Linares \& Geizzelez, 2016; Gil et al., 2011).

IPSA SCIENTIA - Vol. 4 № $1-2019$ 


\section{Comunicación universitaria}

La comunicación en la institución universitaria se encuentra influenciada por la estructura cognitiva, personalidad y aspectos sicológicos de los sujetos como: la motivación, estructura social, valores sociales e intereses de los grupos así como también por el contexto social y las relaciones entre los sujetos (Almirón et al., 2015). Entender estos aspectos nos permite interpretar, comprender y afrontar situaciones conflictivas con resultados de éxito en las organizaciones educativas e instituciones universitarias de educación superior.

Ésta comunicación se da en cualquier organización de la siguiente manera: a) Aunque no exista intencionalidad o conciencia de la misma (palabras, expresiones comunicativas e inclusive inactividad) b) mensajes verbales y con contenido que relacionan a los interlocutores. Es decir, toda comunicación implica "relación entre los participantes" (Anderson, 2010) y puede tener carácter dialéctico o hermenéutico según el caso.

\section{Clima organizacional universitario}

El clima organizacional universitario se relaciona con la calidad del medio laboral para el ejercicio profesional en la institución universitaria y contempla elementos tales como: a) infraestructura física b) características demográficas de personas y grupos (raza, edad, sexo, estarto social, capacidad intelectual, misión y visión de la organización universitaria) c) características propias de la interacción organizacional (liderazgo, competitividad, metas, cooperativismo institucional, sanciones, toma de decisiones) d) valores sociales, estructura cognitiva de los individuos y la organización (cooperación, confianza, honestidad, lealtad, responsabilidad, pertinencia, co-participación en el cumplimiento de metas, normatividad institucional, expectativas grupales e individuales entre otras) (Linares \& Geizzelez, 2016; Araneda et al., 2016).

Representa en términos generales el ambiente de trabajo, la atmósfera que perciben los integrantes de la institución y la inter-relación que se dá entre las estructuras de poder y sus miembros. Es un aspecto capaz de incidir en el estilo de vida, comportamiento y proceso productivo tanto de la institucional como de los miembros que la conforman. Su efecto se puede apreciar en el rendimiento estudiantil, desempeño laboral de docentes y profesionales universitarios y en la calidad de la educación que se imparte en las unidades académicas en donde los logros, innovación, investigación y desarrollo universitario se refleja en las metas alcanzadas por cada uno de sus departamentos (Basham, 2010).

Conocer el clima organizacional de la institución permite trabajar en pro de la mejora de la calidad de la institución universitaria desde las siguientes dimensiones: a) la identificación del clima laboral organizacional b) diagnóstico del clima organizacional vigente en la institución y c) la intervención en la institución con el ánimo de mejorar el clima organizacional de la Universidad superando las no conformidades. Este conocimiento permitirá fortalecer el débil acoplamiento interno entre los diferentes departamentos de la Universidad (Linares \& Geizzelez, 2016).

IPSA SCIENTIA - Vol. 4 № $1-2019$ 


\section{INNOVACIÓN Y DESARROLLO PROFESIONAL EN LA UNIVERSIDAD}

Según Delors, Jacques “....de la educación depende en gran medida el progreso de la humanidad... Hoy más que nunca la educación representa uno de los elementos más poderosos de los que disponemos en la sociedad para forjar nuestro propio futuro.... El principal peligro, en un mundo marcado por la interdependencia y la mundialización, es que se genere un abismo entre una minoría (capaz de moverse en ese mundo nuevo) y una mayoría impotente para desempeñarse en ese destino colectivo" (Delors et al., 1996). Desde esta perspectiva, nuestro norte en el siglo XXI debe enmarcarse en los procesos de innovación, competitividad y globalización soportados desde las Universidades como centros de desarrollo tecnológico y de nuevo conocimiento con acoplamiento débil según los criterios de Weick (1976) para este tipo de instituciones.

La innovación y el desarrollo profesional tanto de docentes, administrativos, directivos y estudiantes debe ser el norte de cualquier institución universitaria con el ánimo de cumplir la misión suprema de cualquier universidad "servirle al ser humano y a la sociedad". El desarrollo profesional de los docentes debe ser más dinámica ya que se ha detectado deficiencia en la formación pedagógica de los mismos, en la red de formadores docentes, en el sistema de promoción profesional ocasionando ansiedad e incertidumbre en los mismos sobre todo a comienzos de su tarea o actividad docente. El trabajo en equipos docentes o de investigación permite a los docentes integrarse de manera más eficaz a los grupos de investigación, semilleros, oficinas, institutos y departamentos (Dávila et al., 2015).

La propuesta de mejora, innovación y desarrollo tecnológico debe ser más dinámica, audaz y contextualizada con el objeto de integrarla de manera eficaz a la economía globalizada con resultados tangibles y con el objeto de ser evaluada a corto, mediano y largo plazo con el ánimo de promover la participación de toda la comunidad universitaria en el diseño y propuesta de proyectos innovadores (Delgado \& Delgado, 2003).

\section{Liderazgo como eje central de desarrollo en la dirección Universitaria}

En la actualidad en las Universidades se ha venido confundiendo los conceptos de autoridad, líder y liderazgo, los cuales en la mayoría de los casos son atribuidos al personal directivo mediante mecanismos formales en virtud del cargo que desempeñan en la organización y según su estructura organizativa. Es necesario tener muy en cuenta que el liderazgo es una capacidad que puede estar presente en cualquier miembro de la organización (liderazgo disperso o distribuido) y como tal debe ser aprovechada con el objeto de abolir la excesiva linealidad con que se lleva a cabo el liderazgo y autoridad en las organizaciones de manera que se construya en niveles múltiples y en torno a valores compartidos. Por esto, la tríada: influencia, grupo y metas debe ser articulada de manera efectiva a la cultura organizativa con el propósito de articularla a una visión compartida. El liderazgo instructivo o pedagógico no debe ser jalonado siempre por la alta dirección sino por todo el personal directamente implicado en los procesos académicos y administrativos como rectores, directores, docentes, estudiantes y centrado específicamente en los resultados de aprendizaje, que motive una dinámica pedagógica/andragógica continua, sostenida y que implique la formulación sostenida de planes de mejoramiento que conduzcan a la generación de

IPSA SCIENTIA - Vol. 4 № $1-2019$ 
proyectos transformadores y que fortalezcan las relaciones con la comunidad universitaria (liderazgo transformador). Según la evolución de los enfoques sobre el liderazgo, podemos decir que en la mayoría de las escuelas, instituciones educativas y universidades actuales, el liderazgo que prevalece es el liderazgo transaccional el cual se caracteriza porque todos los miembros de la organización obedecen a su líder (rector) quien evalúa su liderazgo desde el cumplimiento de las tareas asignadas por él a sus subalternos relacionadas por las metas consagradas en su plan de desarrollo. Este liderazgo se confunde continuamente con las características y cualidades personales de los directivos en donde los rasgos individuales y la eficacia del mismo van de la mano (Delgado \& Delgado, 2003).

Adicionalmente, el enfoque de contingencia o situacional también abunda en las instituciones $u$ organizaciones universitarias ya que este liderazgo se determina por la favorabilidad de la situación o contexto y según Fiedler (1995) los estilos de liderazgo motivado a las relaciones y a la tarea se complementan en el ejercicio diario del líder de hoy en día, ya que aunque el directivo carezca de cualidades de líder son las relaciones y la micropolítica las que condicionan su éxito o fracaso. Estos dos (2) enfoques de liderazgo al que acabamos de referirnos podemos sintetizarlos en el liderazgo directivo (House, 1971), en el que el líder debe ajustar su estilo al contexto y situación laboral en la que se desempeña. En la actualidad, el nuevo liderazgo según Burns (1978) está conformado por un liderazgo político constituido por el liderazgo transaccional y el liderazgo transformacional, el cual se ha venido integrando al nuevo paradigma del nuevo liderazgo en donde se establece una relación líder-seguidores, en donde el primero, ofrece a los segundos un refuerzo que permita alcanzar los compromisos y metas definidas por el líder según los objetivos definidos por la organización para el caso de liderazgo transaccional y en el caso transformacional en donde las aspiraciones de los seguidores van más allá de los propios intereses en beneficio del grupo u organización, aspectos que permean en la actualidad de manera activa a todas las organizaciones y en especial a las instituciones universitarias enmarcadas en el concepto de instituciones débilmente acopladas. Sin embargo, el afirmar que un tipo de liderazgo es propio de una organización no tiene sentido ya que en la actualidad podemos decir que los diferentes enfoques se articulan y complementan unos con otros para constituir lo que hoy conocemos como el "nuevo liderazgo" en donde cada líder según su propio estilo de liderazgo articula a la cultura universitaria sus metas, ajustándose a las nuevas realidades o contextos, gestando una visión compartida e inclusive manipulando la actitud de los miembros de la universidad con el ánimo de transformar sus roles de manera tal que permita construir una organización dinámica y adaptada a las necesidades y condiciones del contexto y que le permite a la universidad, un aprendizaje organizativo que impulsa procesos de innovación y desarrollo tecnológico (Bodla \& Nawaz, 2010).

Es importante tener presente que todos los enfoques sobre liderazgo prestan atención a un solo o único individuo como si poseyera todas las cualidades, actitudes y aptitudes propias de un líder, aspecto que en la nueva perspectiva de mundo globalizado no aplica, es decir, el trabajo en una organización o institución educativa universitaria debe transformar a los miembros de la misma en líderes activos de manera que dicha Universidad se visualice como una "organización de líderes"(independientes del género) que se caracterice por el poder compartido y se asemeje a lo que en la actualidad se conoce como "liderazgo distribuido o distributivo" cuya eficacia se evaluará por las metas, productividad, rendimiento y competitividad sostenida, donde la práctica "colaborativa" se oriente hacia la construcción de una

IPSA SCIENTIA - Vol. 4 № $1-2019$ 
institución universitaria compacta e íntegra caracterizada por la dinámica diaria de sus miembros en donde se cambie el concepto de "poder sobre los otros" por el de "capacidad para actuar con las capacidades de los otros" en donde se visualice una responsabilidad compartida para con los otros (agentes internos y externos a la organización) desde los procesos de gestión de manera que se pueda impulsar una "cultura organizacional" sólida y enfocada hacia el nuevo enfoque de liderazgo en las universidades del siglo XXI (Chaudhuri et al., 2015). El nuevo enfoque de líder debe ser neutral ante la categoría de género y activo ante los retos, metas, misión, visión y en función de la gestión caracterizada por los estilos de liderazgo según el nuevo modelo gerencial de las organizaciones universitarias. Es decir, que en el debate, las diferencias de género en procesos organizativos no deben incidir en la toma de decisiones, orientaciones ni mucho menos en el rol organizativo institucional, o sea que un enfoque centrado en la igualdad de género es deseable (Gil et al., 2011).

Por último, las características ideales de un líder universitario, independiente de su género, deben ser: capacidad de organización y dirección, asertividad, control, gestión, trabajo en equipo, respeto, responsabilidad, humildad, competitividad, independencia, confianza, preocupación por el bienestar de los otros, delicadeza, diplomacia, conciliador, atención tareas, escucha, apoyo, orientación hacia el desarrollo individual y organizacional, orientación hacia el cumplimiento de tareas, orientación hacia la transformación, orientación hacia la innovación, orientación hacia la capacitación del personal, no politización de su liderazgo, excelentes relaciones interpersonales sin perder la autoridad anteponiendo intereses colectivos a intereses personales. Es decir, el nuevo líder universitario debe ser "multi-estilo" y con un alto nivel colaborativo y distributivo con una minimización de los sesgos personales y culturales. Por último, el nuevo líder universitario debe evitar buscar diferencias de género (Harris, 2012; Hernández, 2013) y explorar la forma de unificar el liderazgo haciendo uso de normas de género que impacten en el comportamiento organizativo de la Universidad y que contribuyan constructivamente con el liderazgo distribuido en la universidad permitiendo así dejar atrás la universidad débilmente acoplada para ser transformada en Meta-Universidad (Graves. 1997, Pág. 97).

\section{Conclusiones}

Unificar el liderazgo transaccional y transformacional universitario en un solo liderazgo como el liderazgo directivo con características del nuevo liderazgo de Burns, permitirá transformar la organización universitaria, débilmente acoplada desde la perspectiva de Weick, en Meta-Universidad con altos estándares de calidad y mediada por procesos de investigación, innovación y desarrollo tecnológico que impactaran en procesos empresariales y económicos de la región y el país preparándola hacia los grandes retos de la globalización mundial.

\section{Referencias}

Almirón-Arévalo, V.; A. Tikhomirova; A. Trejo-Toriz y J. García-Ramírez. Liderazgo transaccional vs liderazgo transformacional. (2015). Reidocrea, 4(4), 24-27.

Anderson, Stephen. (2010). Liderazgo Directivo: Claves para una Mejor Escuela. Psicoperspectivas, 9(2), 34-52.

Araneda-Guirriman, Carmen A, Neumann-González, Naomi A, Pedraja-Rejas, Liliana M, \& Rodríguez-Ponce, Emilio R. (2016). Análisis Exploratorio de las Percepciones sobre los Estilos de Liderazgo de los Directivos Universitarios en el Norte de Chile. Formación universitaria, 9(6), 139-152.

IPSA SCIENTIA - Vol. 4 № 1 - 2019 
Basham, L. M. Transformational and transactional leaders in higher education. (2010). International Review of Business Research Papers, 6(6), 141-152.

Bodla, M. A. \& Nawaz, M. M. (2010). Comparative study of full range leadership model among faculty members in public and private sector higher education institutes and universities. International Journal of Business and Management, 5(4), 208-214.

Burns, James M. (1978), Leadership. Harper y Row, New York.

Chaudhuri, M. R.; J. Kettunen y P. Naskar. (2015). Reflections on Leadership Styles from Higher Education in India. Universal Journal of Management, 3(10), 395-401.

Dávila Quintana, C. Delia, \& Mora, José-Ginés, \& Pérez Vázquez, Pedro José, \& Vila, Luis Eduardo (2015). ¿Es posible potenciar la capacidad de liderazgo en la universidad?. INNOVAR. Revista de Ciencias Administrativas y Sociales, 25(56),129-140.

Delgado Torres, Nora A., \& Delgado Torres, Dolores (2003). El líder y el liderazgo: reflexiones. Revista Interamericana de Bibliotecología, 26(2),75-88.

Delors, J., Al Mufti, I., Amagi, I., Carneiro, R., Chung, F., Geremek, B., \& Nazhao, Z. (1996). Informe a la Unesco de la comisión internacional sobre la educación para el siglo XXI: La educación encierra un tesoro. Madrid: Santillana, Ediciones UNESCO, 91-103.

Didriksson, A. (2004). La universidad desde su futuro. Pro-posições, 15(3), 63-73.

Fiedler, F. (1995): "Reflections by an Accidental Theorist". Leadership Quarterly, 6(4), 453-461.

Fong Silva, W., Pitre Redondo, R., Chiquillo Rodelo, J. (2018). Business competitiveness and its association with exogenous factors in plastic recycling companies of the Colombian Caribbean coast. Contemporary Engineering Sciences, 11(2), 71-79.

Fong Silva, W., Colpas Castillo, F., \& Franco Borré, D. (2018). ACADEMIC PERFORMANCE AND ITS ASSOCIATION WITH CLASS ATTENDANCE, INTRINSIC MOTIVATION AND GENDER IN ENGINEERING STUDENTS. IPSA SCIENTIA: Revista Científica Multidisciplinaria, 3(1), 10-16.

Fong Silva, W., Curiel Gómez, R., \& Brito Carrillo, C. (2017). APRENDIZAJE SIGNIFICATIVO Y SU RELACIÓN CON LA MOTIVACIÓN INTRÍNSECA, ESCUELA DE PROCEDENCIA Y ESTRATEGIAS COGNITIVAS EN ESTUDIANTES DE INGENIERÍA. IPSA SCIENTIA: Revista Científica Multidisciplinaria, 2(1), 55-64.

Gil, F., Alcover, C. M., Rico, R., \& Sánchez-Manzanares, M. I. R. I. A. M. (2011). Nuevas formas de liderazgo en equipos de trabajo. Papeles del psicólogo, 32(1), 38-47.

Gómez Ortiz, Rosa Amalia. (2008). El liderazgo empresarial para la innovación tecnológica en las micro, pequeñas y medianas empresas. Pensamiento \& Gestión, (24), 157-194.

Graves, K. (1997). Teachers as course developers. Cambridge: Cambridge University Press.

Harris, Alma (2012), "Distributed Leadership: Implications for the role of the principal", Journal of Management Development, 31(1), 7-17.

Hernández Yáñez, María Lorena. (2013). Liderazgo académico. Revista de la educación superior, 42(167), 105-131.

IPSA SCIENTIA - Vol. 4 № $1-2019$ 
House, R. J. (1971). An path-goal theory of leaders effectiveness, Administrative Science Quarterly, 16, 321-338.

La Rotta, D., Usuga, O. C., \& Clavijo, V. (2020). Perceived service quality factors in online higher education. Learning Environments Research, 23(2), 251-267.

Linares-Morales, J., \& Geizzelez-Luzardo, M. (2016). POLÍTICAS CIENTÍFICAS Y TECNOLÓGICAS PARA LA GESTIÓN DEL CONOCIMIENTO EN INSTITUCIONES UNIVERSITARIAS. IPSA SCIENTIA: Revista Científica Multidisciplinaria, 1(1), 10-17.

Maureira, Oscar, Moforte, Carla, \& González, Gustavo. (2014). Más liderazgo distribuido y menos liderazgo directivo: Nuevas perspectivas para caracterizar procesos de influencia en los centros escolares. Perfiles educativos, 36(146), 134-153.

Mejías Rodríguez, R. (2013). Preparando el camino para una educación abierta. Revista Cubana de Informática Médica, 5(1), 13-19.

Morioka, Sandra Naomi, Iritani, Diego Rodrigues, Ometto, Aldo Roberto, \& Carvalho, Marly Monteiro de. (2018). Systematic review of the literature on corporate sustainability performance measurement: a discussion of contributions and gaps. Gestão \& Produção, 25(2), 284-303.

Muñoz Rojas, D., Vargas Ortiz, L., \& Latta Arias, C. (2018). ESTRUCTURA DEL CLIMA ORGANIZACIONAL EN ALGUNAS UNIVERSIDADES PÚBLICAS DEL NORTE DE COLOMBIA. IPSA SCIENTIA: Revista Científica Multidisciplinaria, 3(1), 17-22.

Orton, J. D., \& Weick, K. E. (1990). Loosely coupled systems: A reconceptualization. Academy of management review, 15(2), 203-223.

Pons Verdú, Fernando J., \& Ramos López, José. (2012). Influencia de los estilos de liderazgo y las prácticas de gestión de RRHH sobre el clima organizacional de innovación. Revista de Psicología del Trabajo y de las Organizaciones, 28(2), 81-98.

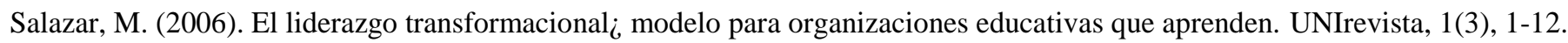

Siboni, B., Nardo, M. T. y Sangiorgi, D. (2013). Italian State University Contemporary Performance Plans: An Intellectual Capital Focus?, Journal of Intellectual Capital, 14 (3), 414-430.

Ugalde, F. V. (1987). ¿ Hacia un nuevo liderazgo sociopolítico? Ensayo sobre la convocatoria social de los empresarios. Estudios sociológicos, 5(15), 433-454.

Waddock, S. A., \& Graves, S. B. (1997a). Quality of management and quality of stakeholder relations. Business and Society, 36(3), 250-279.

Waddock, S. A., \& Graves, S. B. (1997b). The corporate social performance-financial performance link. Strategic Management Journal, 18(4), 303-319.

Wall, T. D., Michie, J., Patterson, M., Wood, S. J., Sheeran, M., Clegg, C. H., \& West, M. (2004). On the validity of subjective measures of company performance. Personnel Psychology, 57(1), 95-118.

Weick, K. E. (1976). Educational Organiza-tions as Loosely Coupled Systems. Administrative Science Quarterly, 21(1), 1-19.

IPSA SCIENTIA - Vol. 4 № 1 - 2019 